\title{
In situ x-ray diffraction of fast compressed iron: Analysis of strains and stress under non-hydrostatic pressure
}

\author{
Zuzana Konôpková, ${ }_{1}^{1}$ André Rothkirch, ${ }^{1}$ Anil K. Singh, ${ }^{2}$ Sergio Speziale, ${ }^{3}$ and Hanns-Peter Liermann ${ }^{1}$ \\ ${ }^{1}$ DESY Photon Science, Notkestrasse 85, D-22602, Hamburg, Germany \\ ${ }^{2}$ Materials Science Division, National Aerospace Laboratories, Council of Scientific and Industrial Research, Bangalore, India \\ ${ }^{3}$ GFZ German Research Centre for Geosciences, Telegrafenberg, 14473 Potsdam, Germany
}

(Received 25 November 2014; published 7 April 2015)

\begin{abstract}
Series of high-pressure x-ray diffraction patterns of iron and its high-pressure polymorphs were collected with 0.1-0.2-s exposure time utilizing a membrane diamond anvil cell (DAC) for compression at various loading and unloading rates to a maximum pressure of $70 \mathrm{GPa}$. Strain rates of $10^{-2} \mathrm{~s}^{-1}$ at a maximum pressurization rate of $4.1 \mathrm{GPa} / \mathrm{s}$ were achieved in non-hydrostatic compression of hcp Fe. Linewidth analysis was used to retrieve strain and uniaxial stress of $\mathrm{Fe}$ as a function of pressure upon both compression and decompression. Analysis of the lattice parameters ratio $c / a$ of hcp Fe indicates the presence of complex non-hydrostatic stress states, which developed as a function of strain rate, relaxation time, and various levels of hydrostaticity. Our results emphasize the importance of a controlled pressurization in DACs because the experimental loading rate strongly influences the stress state of the sample, particularly on decompression. Our time-resolved x-ray diffraction of the phase transition from bcc Fe to hcp Fe reveals residual grains of bcc Fe capable of surviving to very high pressures $(>35 \mathrm{GPa})$ for a few minutes after the transition.
\end{abstract}

DOI: 10.1103/PhysRevB.91.144101

PACS number(s): 61.05.cp, 91.60.Gf

\section{INTRODUCTION}

Static compression experiments in the diamond anvil cell (DAC) at third-generation synchrotrons have yielded a substantial amount of information on structure of matter at high-pressure and simultaneous high-pressure and temperature conditions. Considerable efforts were made to create quasihydrostatic conditions in order to avoid formation of large stresses in the sample, which could bias interpretation of the diffraction patterns, and/or to mimic quasihydrostatic conditions in the Earth's interior as closely as possible. However, not all geological environments on the Earth or extraterrestrial planets are static. For instance, shearing in the subduction zones or meteorite and asteroid impacts are highly dynamic as well as extremely non-hydrostatic, creating different stress-strain fields. While highly dynamic conditions of a meteorite/asteroid impact can be simulated in a gas gun or laser-shock experiments, which are capable of creating strain rates on the order of $10^{4}$ to $10^{7} \mathrm{~s}^{-1}$ [1], less dynamic environments can be simulated in a fast compressed DAC. Recently, both membrane-driven DAC (mDAC) and dynamically driven $\mathrm{DAC}(\mathrm{dDAC})$ techniques have been enhanced to bridge the gap between quasistatic conditions and dynamic shock loading by reaching strain rates in the range of $10^{-2}-10^{2} \mathrm{~s}^{-1}$ [2-5].

While techniques for creating low and moderate strain rates in the DAC have become available [2-5], probes for determining changes in material structure during such fastcompression experiments are lacking. This is because of either insufficient x-ray photon flux (brilliance) or the absence of fast detectors efficient at higher $\mathrm{x}$-ray energies $(>30 \mathrm{keV})$ to conduct $\mathrm{x}$-ray diffraction (XRD) studies with subsecond time resolution.

The advent of new third-generation light sources with unprecedented brilliance such as PETRA III in Hamburg, Germany, and the introduction of fast area detectors optimized for high-energy $\mathrm{x}$-ray diffraction such as the PerkinElmer XRD1621 [6] have made it possible to conduct time-resolved angle-dispersive XRD experiments on continuously compressed materials in DAC with a time resolution of tens of milliseconds. Here, we present a pilot study on the fast compression of iron in order to explore the maximum pressurization (strain) rates that can be realized in mDAC and its effect on the compressional behavior of $\mathrm{Fe}$ under different levels of non-hydrostaticity. Iron has been chosen as the system of interest because of a wealth of studies on its equation of state (EoS) to megabar pressures [7-9], phase diagram [10,11], lattice strain, stress, strength, elasticity, texture, and deformation analysis [12-15] under pressure conducted using static compression in DACs. Phase transition from bcc iron $(\alpha-\mathrm{Fe})$ to the hcp structure ( $\epsilon-\mathrm{Fe})$ and compression of $\epsilon$-Fe up to $70 \mathrm{GPa}$ have been tracked by continuous $\mathrm{x}$-ray diffraction acquisition with 100- and 200-ms time resolution. The maximum rate of pressure increase achieved in $\epsilon-\mathrm{Fe}$ is $\sim 4.1 \mathrm{GPa} / \mathrm{s}$. Using diffraction peak breadths of $\epsilon$-Fe, we have derived the lattice strain and uniaxial stress component $t$, which is a measure of strength, as a function of pressure.

\section{EXPERIMENT}

All compression experiments were performed in a symmetric piston-cylinder-type DAC that was equipped with $0.30-\mathrm{mm}$ culets. Commercial high-purity iron powder (99.98\%, Sigma-Aldrich) with grain size less than $0.01 \mathrm{~mm}$ was loaded without any pressure medium into a rhenium gasket that was indented to $0.03-0.04-\mathrm{mm}$ thickness and drilled to create a $0.10-\mathrm{mm}$-wide sample chamber. A couple of quasihydrostatic experiments were also performed for comparison in which neon served as a pressure-transmitting medium.

Iron was continuously compressed in the DAC equipped with a membrane attached to its back side [16,17]. Pressure in the gas membrane was controlled by a pressure controller from Sanchez Technology (APD200). The pressurization rate and hence the strain rate on the sample were regulated by 
changing the speed of pressure increase on the helium gas within the membrane. During the loading and unloading at different rates, diffraction patterns were continuously recorded with 100- or 200-ms exposure time. The XRD experiments were performed at the Extreme Conditions Beamline (ECB) P02.2 at PETRA III at DESY, Hamburg, Germany. An X-ray beam with a wavelength of $0.29 \AA$ was focused by Kirkpatrick-Baez mirrors down to less than 2 (vertical) $\times$ 2 (horizontal) $\mu \mathrm{m}^{2}$. The sample-detector distance as well as correction for instrumental broadening was calibrated using a $\mathrm{CeO}_{2}$ standard from NIST (SRM 674a) using FIT2D [18]. All 12 compression runs (Table I) started with bcc iron at $0-5$ GPa and continued through the bcc to hcp transition deep into the stability region of the hcp iron up to 30-70 GPa, which was then followed by decompression. Fast collection of data enabled by the PerkinElmer XRD 1621 detector resulted in up to $10000 \mathrm{XRD}$ images, which required development of a data management and data processing strategy, in particular batch processing and optimized peak fitting routines. This was realized by adjusting the $2 \theta$ range for fitting of the data of frame $n+1$ based on the results obtained from the fitting of frame $n$, i.e., through a sliding window of fixed width for each peak. This approach ensured that shifts in peak position could be larger than distances between adjacent peaks. The procedure also implied selecting and interfacing an appropriate program for refinement. The program REFINE (version 2.1) [19] was chosen and moderately modified. Peak positions obtained from fitting and their corresponding Miller indices were processed with this program for refinement of unit-cell parameters.

\section{DATA ANALYSIS}

A solid sample compressed in a DAC develops a stress state that is axially symmetric about the load axis, with the axial stress component $\sigma_{33}$ (parallel to the diamond anvil's axes) being greater than the radial component $\sigma_{11}$. The difference $\left(\sigma_{33}-\sigma_{11}\right) \equiv t$ is called uniaxial stress or differential stress [20] and equals the yield strength of the sample material under a confining pressure $P=\left(\sigma_{33}+2 \sigma_{11}\right) / 3$. The diffraction data obtained with radial diffraction geometry have been used by several investigators to measure $t$ as a function of pressure (e.g., [14,21-24]). Attempts have also been made to obtain strength from the linewidth analysis. In many studies $[25,26]$ strength has been taken as the product of microstrain determined from the linewidth analysis and the Young's modulus. An effort was made [27,28] to develop a rigorous approach based on earlier theories for the ambientpressure linewidth analysis [29]. Defined as the full width (in radians) at half maximum amplitude of the diffraction profile on the $2 \theta$ scale, $2 w$ is related to the "apparent" microstrain $\eta$ and coherent scattering domain size $D$ (often referred to as crystallite size) by

$$
\left(2 w_{h k l} \cos \theta_{h k l}\right)^{2}=(\lambda / D)^{2}+\eta_{h k l}^{2} \sin ^{2} \theta_{h k l} .
$$

The strength $t$ is given by

$$
t \cong\left\langle\eta_{h k l}\right\rangle E / 2
$$

The symbol $\langle\cdot\rangle$ denotes the average over all the recorded reflections, and $E$ is the aggregate Young's modulus. The agreement between the strength derived from the linewidth using the above equation and that obtained from the line-shift analysis of the radial diffraction data in many cases $[13,27,30,31]$ provided support for Eqs. (1) and (2).

Peak profile fitting was carried out on all diffraction patterns collected during the loading-unloading cycle using a Gaussian peak shape that yielded data on peak positions and linewidth (FWHM, 2w). Typically, six to seven reflections (110, 200, $211,220,310,222,321)$ of the bcc Fe were included in the analysis, whereas only three to four reflections $(100,101,102$, 110) of the hcp phase were involved due to overlapping of the peaks or weak intensities of reflections at higher diffraction angles. The FWHM values of the sample were corrected for instrumental broadening based on instrument response to $\mathrm{CeO}_{2}$ standard. The corrected FWHM values along with the $2 \theta$ values were used to construct the $(2 w \cos \theta)^{2}$ vs $\sin ^{2} \theta$ plots, in which the slope determines the microstrain $\eta$ and the intercept yields the grain size $D$. We focus here mainly on the determination of the microstrain from the slope of the linear regression fits.

\section{RESULTS}

Figure 1 shows a representative data set of a fastcompression experiment (run 6) in the form of a contour plot that displays all integrated diffraction patterns (about 1000 files). The patterns are stacked on top of each other as time progresses from the bottom to the top. The inset in Fig. 1 depicts time variation of the gas pressure in the membrane (right vertical axis, green plot) and the sample pressure response (left vertical axis, purple plot). The apparent pressure in the sample was calculated from the $d$ spacings using the equation of state of $\alpha-\mathrm{Fe}$ [32] ( $K=159 \mathrm{GPa}$, $\left.K^{\prime}=4\right)$ and $\epsilon$-Fe [8] $\left(K=165 \mathrm{GPa}, K^{\prime}=4.97\right)$. The first 120 patterns follow compression of bcc iron, after which

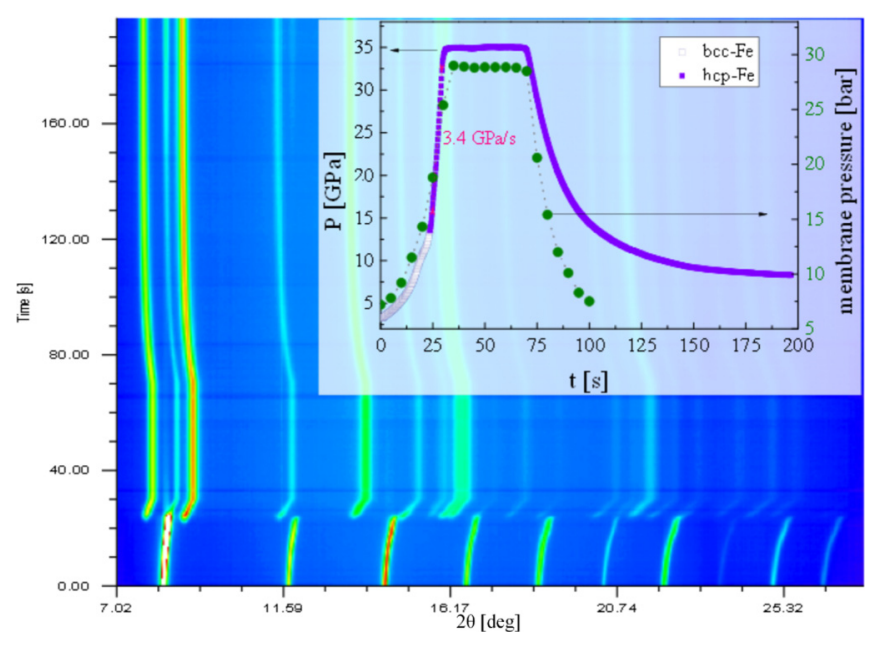

FIG. 1. (Color online) Contour plot of the two-dimensional integrated diffraction patterns depicting phase transition from $\alpha$ to $\epsilon$ iron, run 6 . The inset shows pressure in the membrane (right axis) and in the sample (left axis) as a function of time. Diffraction patterns were collected continuously with 200-ms acquisition steps. The entire data collection time lasted no longer than 200 s. Approximately 1000 diffraction images were collected. 
TABLE I. Summary of all fast-compression runs.

\begin{tabular}{|c|c|c|c|c|c|c|}
\hline Run & $\begin{array}{l}\text { Pressure } \\
\text { medium }\end{array}$ & $\begin{array}{c}\text { Pressure rate } \\
\text { membrane (bars/min) }\end{array}$ & $\begin{array}{c}\text { Pressure rate } \\
\text { hcp Fe }(\mathrm{GPa} / \mathrm{s})\end{array}$ & $\begin{array}{l}\text { Maximum } P \\
\quad(\mathrm{GPa})\end{array}$ & $\begin{array}{c}\text { Minimum } P \\
\text { after decompression }(\mathrm{GPa})\end{array}$ & $\begin{array}{l}\text { Exposure } \\
\text { time }(\mathrm{ms})\end{array}$ \\
\hline 1 & none & 2.5 & 0.09 & 62 & 8 & 200 \\
\hline 2 & none & 10 & 0.4 & 63 & 16 & 200 \\
\hline 3 & none & 20 & 0.7 & 66 & 28 & 200 \\
\hline 4 & none & 40 & 1 & 23 & 4 & 200 \\
\hline 5 & none & 50 & 3 & 40 & 12 & 200 \\
\hline 6 & none & 80 & 3.4 & 35 & 8 & 200 \\
\hline 7 & none & 40 & 4.1 & 66 & 25 & 100 \\
\hline 8 & none & 60 & 3.1 & 57 & 20 & 100 \\
\hline 9 & $\mathrm{Ne}$ & 2.5 & 0.02 & 22 & 5 & 200 \\
\hline 10 & $\mathrm{Ne}$ & 10 & 0.2 & 24 & 14 & 200 \\
\hline 11 & $\mathrm{Ne}$ & 20 & 0.6 & 51 & 20 & 200 \\
\hline 12 & $\mathrm{Ne}$ & 40 & 0.7 & 34 & 11 & 200 \\
\hline
\end{tabular}

iron transforms to the hcp crystal structure. After further compression of $\epsilon-\mathrm{Fe}$, the gas pressure in the membrane is kept constant, and the decompression cycle follows. Although the unloading rate on the membrane is the same as during compression, the real unloading of the sample is somewhat slower and incomplete due to friction between the piston and cylinder of the symmetric type DAC.

The pressurization rate of $3.4 \mathrm{GPa} / \mathrm{s}$ was estimated from the pressure calculated using the equation of state of iron after the transformation into the hcp phase until the maximum pressure was reached (linear regression through the pressure-time plot). The corresponding strain rate is $\sim 10^{-2} \mathrm{~s}^{-1}$. Several data sets with different loading rates were obtained by regulating the gas flow in the membrane. The resulting pressurization rates in the sample varied from 0.09 to $4.1 \mathrm{GPa} / \mathrm{s}$ (see Table I). We also conducted a few compression runs under quasihydrostatic conditions using neon as a pressure-transmitting medium (Fig. 2).

Peak positions and corresponding widths (FWHM) of all diffraction patterns were used to construct the $(\mathrm{FWHM} \cos \theta)^{2}$ vs $\sin ^{2} \theta$ plots. A linear regression of these plots yields slope values that are representative of microstrain. Figure 3 depicts $\eta$ during the slowest pressurization rate $(0.09 \mathrm{GPa} / \mathrm{s}$, run 1$)$ and the fastest pressurization rate $(4.1 \mathrm{GPa} / \mathrm{s}$, run 7$)$. These data sets consist of approximately 10000 and 2500 data points, respectively. The insets illustrate the variation of the nominal sample pressure (right axis) and $\eta$ (left axis) versus time.

The uniaxial stress component $t$ is of great importance as it provides a lower bound on the yield stress and strength of the material. It can be evaluated using the microstrain values from the linewidth analysis described above and Young's modulus $E$, which has been computed from shear $(G)$ and bulk $(K)$ moduli using the relation $E=9 K G /(3 K+G)$. The bulk modulus has been calculated from the EoS of $\epsilon$-Fe [8]. The shear modulus has been determined based on the recent nuclear resonant inelastic x-ray scattering experiments [15]. The resulting Young's modulus to $60 \mathrm{GPa}$ can be described as $E=218.18+4.74 P$. The uniaxial stress component $t$ of $\epsilon-\mathrm{Fe}$ as a function of pressure is shown in Fig. 4 for the same data sets as presented in Fig. 3. The linewidth analysis has also been applied to one of the quasihydrostatic runs (run 11). As expected, the resulting uniaxial stress is much lower due to the presence of soft neon.
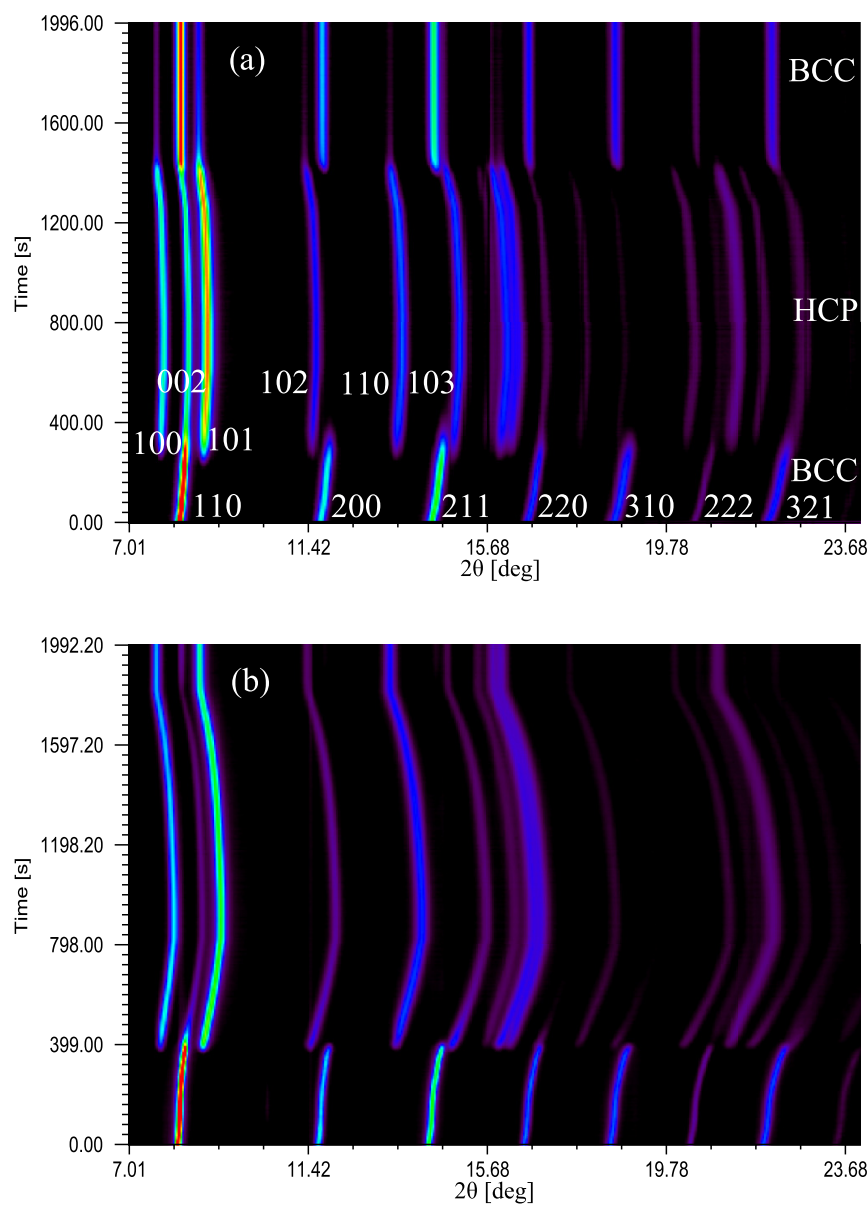

FIG. 2. (Color online) Contour plot of compression with (a) hydrostatic pressure medium (neon), run 9, and (b) no pressure medium, run 1 . The effect of the pressure medium on development of the texture is clearly observed; that is, without the use of neon, the intensity of the 002 reflection of $\epsilon$-Fe is significantly reduced. 

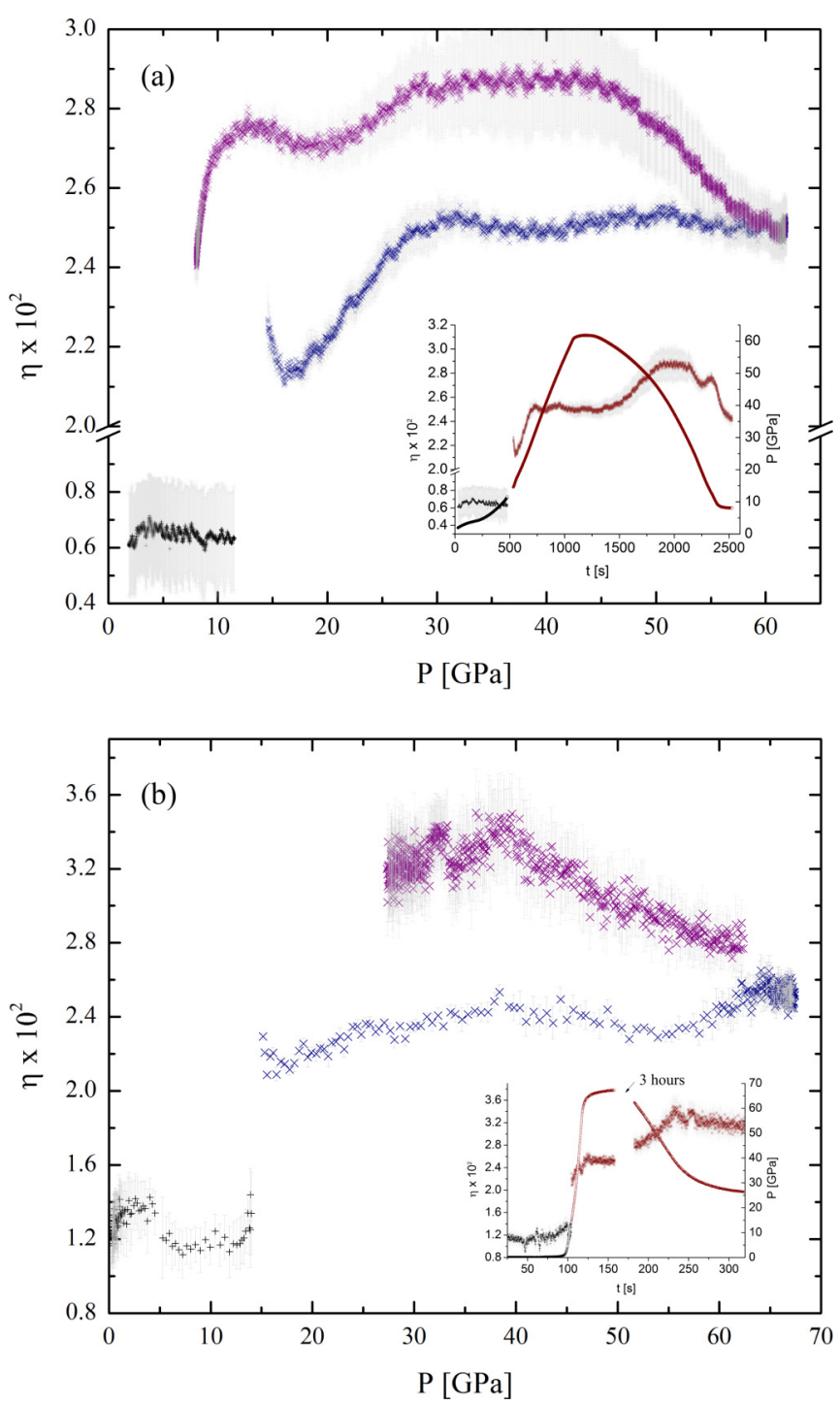

FIG. 3. (Color online) Microstrain in $\alpha$-Fe (black plus signs) and $\epsilon$-Fe (blue crosses: compression, purple crosses: decompression) as a function of pressure. The gray areas denote errors of the regression fits to the $[\mathrm{FWHM} \cos (\theta)]^{2}$ vs $\sin ^{2} \theta$ data (the standard error of the linear fit is represented here by a plus-minus error bar). (a) Run 1 $(0.09 \mathrm{GPa} / \mathrm{s})$ and $(\mathrm{b})$ run $7(4.1 \mathrm{GPa} / \mathrm{s})$. Insets show the evolution strain/pressure vs time.

\section{DISCUSSION}

\section{A. Microstrain}

Microstrain $\eta$ was calculated for all compression experiments. It shows similar behavior as a function of pressure in all the experiments. Thus, we show and discuss the evolution for only the slowest (run 1) and the fastest (run 7; Fig. 3) compression experiments. The insets in Fig. 3 display the time evolution of the sample pressure and microstrain; in run 7, the pressure in the membrane was kept constant for $3 \mathrm{~h}$ (called the relaxation period in the following) after the ramping phase, after which the sample was decompressed. The microstrain $\eta$ is fairly constant across the $\alpha$-Fe stability field; however, an abrupt increase in microstrain accompanies the phase transition to $\epsilon$-Fe. Microstrain continues to increase in

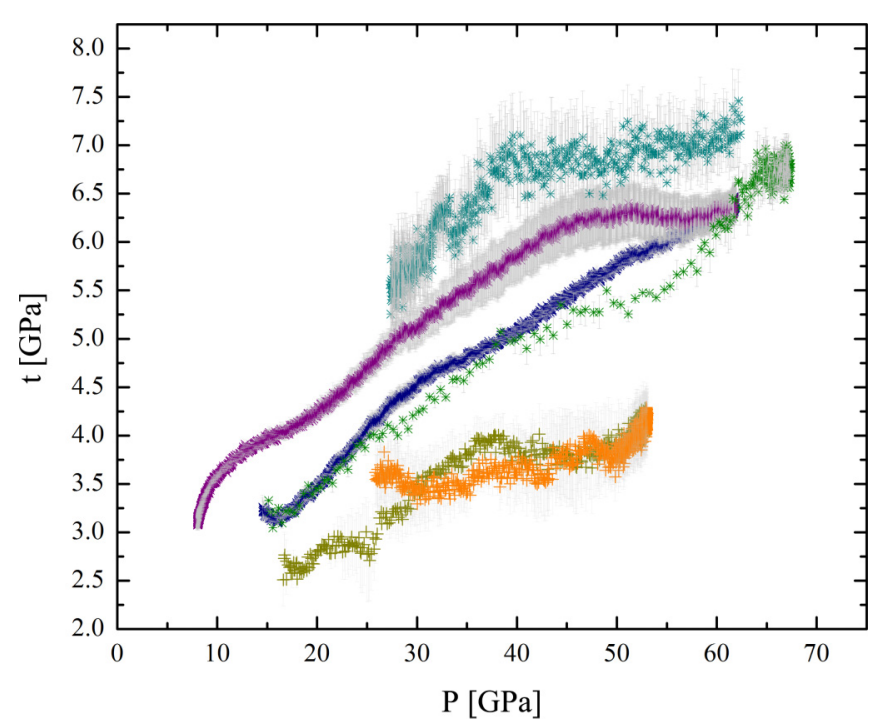

FIG. 4. (Color online) Uniaxial stress component of the $\epsilon$-Fe calculated for run 1 (blue: compression, purple: decompression), run 7 (dark green: compression, dark cyan: decompression) and quasihydrostatic run 11 (dark yellow: compression, orange: decompression). The error bars were propagated from the estimated uncertainties on uniaxial strain.

the $\epsilon$-Fe stability field until a pressure of $30 \mathrm{GPa}$, after which it remains constant at a value of $0.024-0.025$ even though pressure in the sample increases further to more than $60 \mathrm{GPa}$. The magnitude of microstrain is higher than observed in the static compression study of $\epsilon$-Fe $(\sim 0.02)$ [13]; however, it is consistent with the observation that microstrains become pressure independent above $30 \mathrm{GPa}$.

Linewidth analysis has not been previously applied in detail to the decompression of a sample in the DAC. On decompression, $\eta$ increases, peaks at $\sim 40 \mathrm{GPa}$, and stays constant to a pressure of $\sim 30 \mathrm{GPa}$, after which it begins to decrease. The increase of $\eta$ during the decompression is common to all data sets independent of the pressurization rates. The magnitude of microstrain appears to be dependent on the rate (higher values of $\eta$ were obtained upon decompression in the run with a larger pressurization rate). In general, the pressure dependence of microstrain can be interpreted as follows: after an initial flowing and thinning of the gasket and the sample, both become locked between the cupped diamonds [33], and the strain stops increasing. Only during decompression are the gasket and the sample able to flow again, hence enabling the strain to increase even further.

It is interesting to note that in decompression $\epsilon$-Fe remains stable down to at least $8 \mathrm{GPa}$, yet there is a distinct drop in $\eta$ values at a pressure of about $13 \mathrm{GPa}$ and below, possibly indicating the beginning of reverse transformation. A closer examination of Fig. 1 reveals that the intensity of the 002 reflection of $\epsilon$-Fe (it overlaps with the 110 reflection of $\alpha-\mathrm{Fe})$ increases, and very weak bcc lines appear at around $150 \mathrm{~s}(\sim 11 \mathrm{GPa})$ that are not obvious in single integrated diffraction patterns. Reverse transformation manifested by a sudden increase in the peak height at pressures comparable to the transformation pressure during compression have been documented already by Huang et al. [34]. 


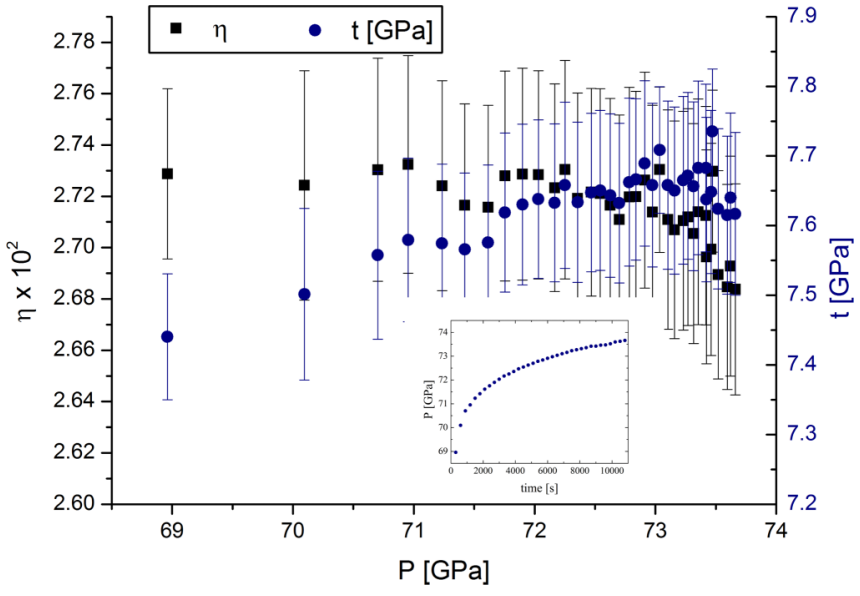

FIG. 5. (Color online) Strain (black squares, left axis) and stress (blue dots, right axis) as a function of pressure during the 3-h relaxation period in run 7 . Pressure variation with time is shown in the inset.

The uniaxial stress component $t$, or compressive yield strength of $\epsilon$-Fe, increases linearly with pressure at nearly the same rate in all the runs (see, for instance, runs 1 and 7 in Fig. 4). Larger values of $t$ are, however, observed upon decompression in the samples subjected to higher pressurization rates. Interestingly, samples subjected to higher loading/unloading rates experience higher stresses at lower pressures during decompression.

During the 3 -h relaxation period in run 7 , diffraction patterns with 10 -s exposure time were collected every 5 min. Analysis of the diffraction data reveals that the pressure continued to rise during this period by almost $5 \mathrm{GPa}$ (Fig. 5). Strength calculations yield somewhat higher values than those calculated from 100-ms diffraction patterns, which we attribute to better statistics as a result of longer exposure time. Microstrains are initially constant but then start decreasing after the first $2 \mathrm{~h}$; the stress, on the other hand, gradually increases with pressure, reaching a plateau towards the end of the relaxation period. These observations indicate that the time scale for sample, gasket, and diamond stress-strain relaxation is on the order of hours.

Values of strength and microstrain at $62 \mathrm{GPa}$ are somewhat higher than those reported in Singh et al. [13], and the $t$ values are close to the few data points in the lower pressure range reported by Hemley et al. [14]. However, considering the limitations of the method used, the less than optimal intensities of the diffraction peaks, and the small number of peaks involved in the analysis, we do not emphasize the absolute values, but rather the relative evolution of strain and stress as a function of pressure on both compression and decompression, as discussed earlier.

\section{B. Ratio of $c / a$}

The evolution of the ratio of the lattice parameters $c / a$ of $\epsilon$-Fe is illustrated in Fig. 6 as a function of pressure for three non-hydrostatic runs at different pressurization rates and one quasihydrostatic run with neon as a pressure medium. For the runs without any pressure medium, the representative

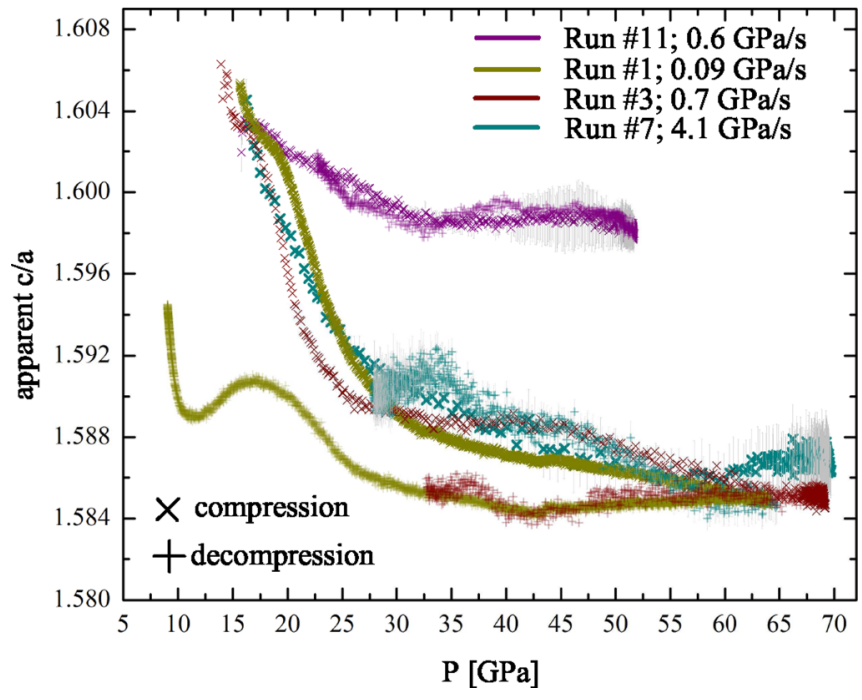

FIG. 6. (Color online) The $c / a$ ratio at different pressurization rates as a function of pressure. Runs 1,3 , and 8 are under nonhydrostatic conditions; run 11 is compression with neon. Crosses denote the compression phase, and plus signs denote decompression.

pressure was taken as the "nominal" pressure (pressure calculated from the $d$ spacings and EoS of $\epsilon-\mathrm{Fe}$ ) corrected by adding $+1 / 3 t$ to account for the uniaxial stresses [13] to be closer to the hydrostatic conditions. Despite this correction, there is a clear discrepancy between the runs that used neon and those that compressed iron without any pressure-transmitting medium. This points to the significance of the non-hydrostatic stress states on apparent lattice parameters calculated from the measured $d$ spacings under different levels of hydrostaticity. In general, the difference has been clearly observed in XRD experiments in the radial configuration $[21,35]$ in which the $\mathrm{x}$-rays probe the sample at a $90^{\circ}$ angle to the compression axis. In radial $\mathrm{x}$-ray diffraction experiments the lattice parameters measured at the angle between the diffracting plane normal and the load axis corresponding to the hydrostatic conditions $\left(\sim 54.7^{\circ}\right)$ are smaller than those obtained from angles that correspond more to the conventional diamond-cell geometry (parallel to the load axis). However, the $c / a$ ratio shows the opposite behavior. For example, Singh et al. [21] compare the lattice parameters of $\epsilon-\mathrm{Fe}$ at $52 \mathrm{GPa}$ at the "hydrostatic angle," $a_{P}$ and $c_{P}$, and at the "conventional DAC geometry angle," $a_{\psi}$ and $c_{\psi}$. The unit-cell volumes calculated from $a_{P}$ and $c_{P}$ are smaller than those calculated from $a_{\psi}$ and $c_{\psi}$; however, $c_{P} / a_{P}=1.5965$, whereas $c_{\psi} / a_{\psi}=1.5938$, i.e., $c_{P} / a_{P}>c_{\psi} / a_{\psi}$. This is consistent with our observation that more hydrostatic conditions result in a higher $c / a$ ratio. Moreover, the sensitivity of the $c / a$ ratio to the stress state is also noticeable if we consider single non-hydrostatic runs. In fact, stress conditions in compression are different from those in decompression, as seen by different apparent $c / a$ ratio in runs 1 and 3 (Fig. 6).

The difference between $c / a$ ratios in hydrostatic and nonhydrostatic conditions is governed by the elastic properties of $\epsilon$-Fe. The effect of uniaxial stress in addition to the hydrostatic part of the stress in the DAC depends on the absolute value of the uniaxial stress and also on the specific elastic constants 
associated with the direction $[h k l]$ perpendicular to the $(h k l)$ lattice plane. Under such conditions, the measured $d$ spacing in the axial XRD geometry is expressed as

$$
d_{m}(h k l)=d_{p}(h k l)[1+Q(h k l)],
$$

where $d_{p}$ is the $d$ spacing corresponding to the hydrostatic conditions and $Q(h k l)$ is, under the Reuss approximation, given by

$$
Q(h k l)=\frac{t}{3} \frac{1}{2 G_{R}(h k l)},
$$

with $G_{R}(h k l)$ being the aggregate shear modulus. Equations (3) and (4) for 100 and 002 reflections then read

$$
\begin{gathered}
d_{m}(100)=\left[1+\frac{t}{3} \frac{1}{2}\left(\frac{3}{E(100)}-\beta(100)\right)\right] d_{p}(100), \\
d_{m}(002)=\left[1+\frac{t}{3} \frac{1}{2}\left(\frac{3}{E(002)}-\beta(002)\right)\right] d_{p}(002) .
\end{gathered}
$$

In the case of a hexagonal system and using the relations for linear compressibility $\beta$ and Young's modulus $E$, expressed in terms of the elastic compliances [36], the ratio of $d_{m}(002) / d_{m}(100)$ (as a proxy of the $c / a$ ratio) becomes

$$
\frac{d_{m}(002)}{d_{m}(100)}=\frac{1+t / 6\left(2 S_{33}-2 S_{13}\right)}{1+t / 6\left(2 S_{11}-S_{12}-S_{13}\right)} \frac{d_{p}(002)}{d_{p}(100)} .
$$

In Eq. (7), $d_{m}(002) / d_{m}(100)$ is determined from the nonhydrostatic compressions, whereas $d_{p}(002) / d_{p}(100)$ is taken from the quasihydrostatic run. At $50 \mathrm{GPa}$,

$$
\frac{d_{m}(002) / d_{m}(100)}{d_{p}(002) / d_{p}(100)}=0.9974 \text {. }
$$

According to Eq. (7), the ratio of $d$ spacings under hydrostatic and non-hydrostatic conditions is dependent on the combination of the elastic compliances $S_{i j}$ and the uniaxial stress $t$. The elastic constants tensor for $\epsilon$-Fe under compression was computed based on the published first-principles calculations [37] and was measured in lattice strain experiments [38]. The elastic constants listed in Table IV in Steinle-Neumann et al. [37] yield typical values of

$$
\frac{1+t / 6\left(2 S_{33}-2 S_{13}\right)}{1+t / 6\left(2 S_{11}-S_{12}-S_{13}\right)}=0.9997
$$

with $t=5.8$ taken from our results at $50 \mathrm{GPa}$. This is in agreement with the experimental observation (Fig. 6). At the same time we observe that $3 / E(002)-\beta(002)$ for the $c$ direction is smaller than that for the $a$ direction, $3 / E(100)-\beta(100)$. The comparison of the result based on our experiments and that based on the elastic tensor predicted by Steinle-Neumann et al. [37] points to much larger elastic anisotropy $\left(C_{33} / C_{11}\right)$ and/or underestimation of $t$ in our experiments. However, the effect of $t$ in Eq. (7) is weak. To reconcile the two results the elastic anisotropy would have to be larger by a factor of 10 in the elastic tensor derived from the computations [37]. The elastic constants tensor reported from radial $\mathrm{x}$-ray diffraction experiments [38] would require anisotropy to increase by a factor of 2.

A significant "softening," i.e., change in the pressure dependence of the $c / a$ ratio, can be observed in all compression runs between 25 and $50 \mathrm{GPa}$. This anomaly has recently been
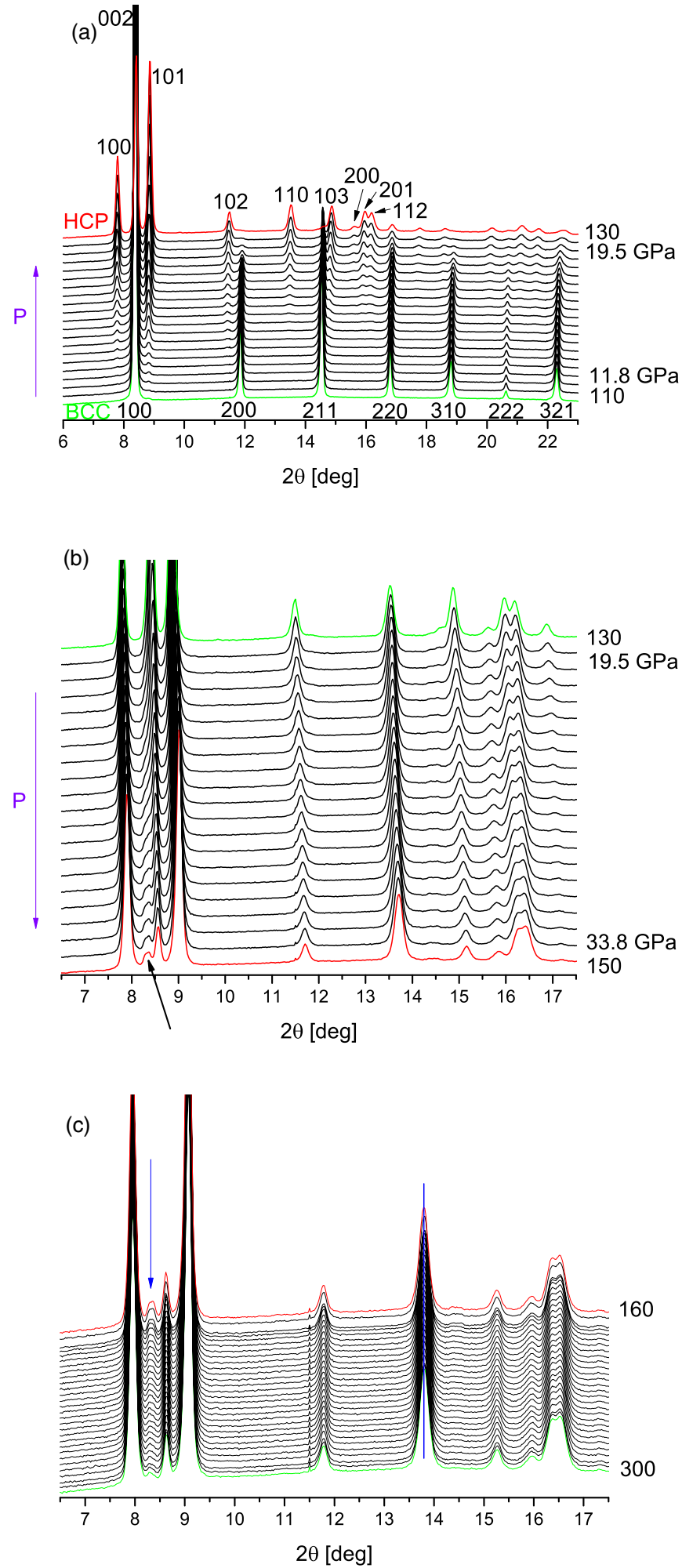

FIG. 7. (Color online) Two-dimensional integrated diffraction patterns for run 6 (a) in the transition region $(\sim 8 \mathrm{GPa}$ increase in $4 \mathrm{~s}$; pressure increases from bottom to top), (b) after the transition region upon further pressure increase ( $14 \mathrm{GPa}$ increase in $4 \mathrm{~s}$; pressure increases from top to bottom), and (c) for pressure stabilization at $35 \mathrm{GPa}$ ( $28 \mathrm{~s}$; time sequence is from top to bottom). Numbers on the right denote the sequence of diffraction patterns and nominal pressure (in GPa). 
claimed to be evidence of the electronic topological transition associated with the change of the Fermi surface [39]. Anomaly features are similar in both hydrostatic and non-hydrostatic runs and can also be observed during the decompression phase in which a slight change in the slope of the $c / a$ ratio occurs at almost precisely $40 \mathrm{GPa}$. The magnitude/character of the anomaly on the decompression appears to be dependent on the pressurization rate and/or maximum pressure reached.

\section{C. $\alpha-\epsilon$ phase transition}

The $\alpha-\epsilon$ transformation in iron is presented in detail as two-dimensional (2D) integrated patterns in Fig. 7 for one of the fastest runs (run 6). Each pattern originates from a 200-ms exposure. The transition begins at $12 \mathrm{GPa}$ and is complete after $4 \mathrm{~s}$ at around $20 \mathrm{GPa}$. During this time bcc and hcp phases coexist. With increasing pressure, the number of hcp grains that grow at the expense of $\alpha$-Fe is evidenced by relative changes in the intensities of bcc and hcp Fe diffraction peaks. It can be observed that in the transition region where the two phases coexist, the diffraction peaks of $\epsilon$-Fe $(100,102,110$, and 200) exhibit shifts towards lower diffraction angles rather than to higher diffraction angles, which would be expected on compression of the sample [Fig. 7(a)]. Metastable structures or structurally distorted interfaces were suggested to cause some structural anomalies during this transformation [40]. After the phase transition is completed, all peaks shift to higher angles as a result of the reduction of the cell parameters, as expected during the pressure increase [Fig. 7(b)]. The mechanism of bcc-hcp transformation has been extensively discussed in Miyagi et al. [41]. It is generally accepted that the transformation is sluggish and completed only at about $22 \mathrm{GPa}$. However, upon further compression, an extra peak becomes visible at around $2 \theta=8.3^{\circ}$. The position of the peak remains constant as pressure increases to more than $35 \mathrm{GPa}$, and its position coincides with the position of the bcc Fe 100 reflection (just before the transformation). In the diffraction image its corresponding ring displays continuous intensities as a function of the azimuthal direction. Similar behavior of the 211 bcc reflection can be identified at about $2 \theta=14.4^{\circ}$, although it has much lower intensity. Both peaks exhibit large broadening in comparison to the hcp lines. During the relaxation period [Fig. 7(c)] the intensity of the remaining bcc Fe peak is decreasing while the intensities of the hcp-phase peaks remain constant. The intensity of the peak significantly diminishes within a minute, which might be a reason why it is not recognized in traditional compression experiments. This peak is not observed in hydrostatic runs with neon. Both observations confirm the complexity of the phase transition from $\alpha$ to $\epsilon$ iron and its sensitivity to stress conditions and pressurization rate [40,41]. Previous studies on the deformation of polycrystalline iron analyzed lattice- preferred orientations of $\alpha$ - and $\epsilon$-Fe using radial diffraction experiments and variation of the diffraction intensities [12,41]. The development of texture in $\epsilon$-Fe in our experiments is clear from the reduced intensity and "spottiness" of the 002 reflection, which has been explained by the tendency of the $c$ axis of $\epsilon-\mathrm{Fe}$ crystals to align in a direction parallel to the compression axis at higher pressures. In $\alpha$-Fe, just before the phase transition, most of the (111) and (100) planes are oriented orthogonal to the compression axis. A combination of these mechanisms, perhaps along with the lattice plane-dependent strength [with basal plane (002) being the weakest in some hcp metals [35]], leads to a large stress "buffer" surrounding the remaining bcc Fe grains that prevents them from immediate transformation. These grains must be in such a configuration that they do not feel the external pressure imposed on them, as seen by the constant $d$ spacing while the overall pressure increases [Fig. 7(c)]. However, they also must experience an extremely heterogeneous stress distribution, as indicated by the broadening of the peaks. Ultimately, it takes a few minutes before the pockets of residual bcc Fe collapse. The transformation of the bcc phase is thus controlled by time in addition to stress (in contrast to the report of Huang et al. [34]). This observation points to the importance of the stress distribution before, during, and after the phase transition for the kinetics of the phase transition itself and could perhaps be better resolved by fast-compression experiments in the radial geometry.

\section{CONCLUSIONS}

This study presents a systematic experimental investigation of real-time XRD acquisition with a subsecond exposure time of a continuously compressed sample in the membrane DAC. Stress and microstrain fields in the iron sample were evaluated under non-hydrostatic and quasihydrostatic conditions. The method combines the diffraction capabilities of brilliant highenergy x-ray diffraction with fast area detectors optimized for high energies and developments in data transfer and processing to handle data sets of up to several thousands of diffraction images.

These recent technical developments enabled us to finely control the compression/decompression path and track the stress/strain conditions of the sample by collecting XRD with a frequency of up to $10 \mathrm{~Hz}$. We have shown that different pressurization rates yielded different strain-stress fields and strongly affect the progress of the bcc to hcp transition in Fe.

\section{ACKNOWLEDGMENTS}

This research was carried out at the light source PETRA III at DESY, a member of the Helmholtz Association (HGF). We thank the reviewers for their comments.
[1] Science and Technology of High Pressure, Proceedings of AIRAPT-17, edited by M. H. Manghnani, W. J. Nellis, and M. F. Nicol, Vol. 1 (Universities Press (India) Limited, Hyderabad, 1999).

[2] W. J. Evans, C.-S. Yoo, G. W. Lee, H. Cynn, M. J. Lipp, and K. Visbeck, Rev. Sci. Instrum. 78, 073904 (2007).
[3] N. Velisavljevic, S. Sinogeikin, R. Saavedra, R. S. Chellappa, A. Rothkirch, D. M. Dattelbaum, Z. Konopkova, H.-P. Liermann, M. Bishop, G. M. Tsoi, and Y. K. Vohra, J. Phys. Conf. Ser. 500, 032020 (2014).

[4] J.-Y. Chen, M. Kim, C.-S. Yoo, H.-P. Liermann, and W. Evans, J. Phys. Conf. Ser. 500, 142006 (2014). 
[5] J.-Y. Chen, C.-S. Yoo, W. Evans, H.-P. Liermann, H. Cynn, M. Kim, and Z. Jenei, Phys. Rev. B 90, 144104 (2014).

[6] XRD 1621 AN/CN - Digital X-Ray Detector, Reference Manual: Digital Imaging, PerkinElmer Optoelectronics, Rev. 2008-0730, PerkinElmer, Inc, Freemont, CA (2008).

[7] H.-K. Mao, Y. Wu, L. C. Chen, and J. F. Shu, J. Geophys. Res. 95, 21737 (1990).

[8] A. Dewaele, P. Loubeyre, F. Occelli, M. Mezouar, P. I. Dorogokupets, and M. Torrent, Phys. Rev. Lett. 97, 215504 (2006).

[9] L. S. Dubrovinsky, S. K. Saxena, F. Tutti, and S. Rekhi, Phys. Rev. Lett. 84, 1720 (2000).

[10] R. Boehler, D. S.-Pérez, D. Errandonea, and M. Mezouar, J. Phys. Conf. Ser. 121, 022018 (2008).

[11] T. Komabayashi and Y. Fei, J. Geophys. Res. 115, B03202 (2010).

[12] S. Merkel, H.-R. Wenk, P. Gillet, H.-K. Mao, and R. J. Hemley, Phys. Earth Planet. Inter. 145, 239 (2004).

[13] A. Singh, A. Jain, H. Liermann, and S. Saxena, J. Phys. Chem. Solids 67, 2197 (2006).

[14] R. J. Hemley, H.-K. Mao, G. Shen, J. Badro, P. Gillet, M. Hanfland, and D. Häusermann, Science 276, 1242 (1997)

[15] A. Gleason and W. Mao, Nat. Geosci. 6, 571 (2013).

[16] W. B. Daniels and M. G. Ryschkevich, Rev. Sci. Instrum. 54, 115 (1983).

[17] R. LeToullec, P. Loubeyre, J. P. Pinceaux, H.-K. Mao, and J. Hu, High Pressure Res. 6, 379 (1992).

[18] A. Hammersley, S. Svensson, M. Hanfland, A. Fitch, and D. Häusermann, High Pressure Res. 14, 235 (1996).

[19] Robert T. Downs, REFINE is part of the software CRYSTALSLEUTH, http://www.geo.arizona.edu/xtal/group/software.htm.

[20] A. Singh, J. Appl. Phys. 73, 4278 (1993).

[21] A. Singh, C. Balasingh, H. K. Mao, R. Hemley, and J. Shu, J. Appl. Phys. 83, 7567 (1998).
[22] S. Merkel, H. Wenk, J. Shu, G. Shen, P. Gillet, H.-K. Mao, and R. Hemley, J. Geophys. Res. 107, 2271 (2002).

[23] A. Kavner and T. Duffy, Geophys. Res. Lett. 28, 2691 (2001).

[24] S. Shieh, T. Duffy, and B. Li, Phys. Rev. Lett. 89, 255507 (2002).

[25] J. Chen, D. Weidner, and M. Vaughan, Nature (London) 419, 824 (2002).

[26] J. Chen, N. Schmid, J. Chen, L. Wang, D. Weidner, J. Zhang, and Y. Wang, J. Mater. Sci. 40, 5763 (2005).

[27] A. Singh, H. Liermann, and S. Saxena, Solid State Commun. 132, 795 (2004).

[28] A. Singh, J. Phys. Chem. Solids 65, 1589 (2004).

[29] J. Langford, J. Appl. Crystallogr. 4, 164 (1971).

[30] A. Singh, H. Liermann, S. Saxena, H. Mao, and S. Devi, J. Phys. Condens. Matter 18, S969 (2006).

[31] A. Singh, D. Andrault, and P. Bouvier, Phys. Earth Planet. Inter. 208-209, 1 (2012).

[32] J. Zhang and F. Guyot, Phys. Chem. Miner. 26, 206 (1999).

[33] S. Merkel, R. J. Hemley, and H.-K. Mao, Appl. Phys. Lett. 74, 656 (1999).

[34] E. Huang, W. A. Bassett, and P. Tao, High-Pressure in Mineral Physics, edited by M. H. Manghnani and Y. Syono (Terra Scientific Publishing Company, Tokyo/American Geophysical Union, Washington, DC, 1987), pp. 165-172.

[35] M. Weinberger, S. Tolbert, and A. Kavner, Phys. Rev. Lett. 100, 045506 (2008).

[36] T. Uchida, N. Funamori, and T. Yagi, J. Appl. Phys. 80, 739 (1996).

[37] G. Steinle-Neumann, L. Stixrude, and R. Cohen, Phys. Rev. B 60, 791 (1999).

[38] A. Singh, H. Mao, J. Shu, and R. Hemley, Phys. Rev. Lett. 80, 2157 (1998).

[39] K. Glazyrin et al., Phys. Rev. Lett. 110, 117206 (2013).

[40] F. Wang and R. Ingalls, Phys. Rev. B 57, 5647 (1998).

[41] L. Miyagi, M. Kunz, J. Knight, J. Nasiatka, M. Voltolini, and H.-R. Wenk, J. Appl. Phys. 104, 103510 (2008). 